\title{
Analysis of CRISPR in Streptococcus mutans suggests frequent occurrence of acquired immunity against infection by M102-like bacteriophages
}

Correspondence

Jan R. van der Ploeg

jan.vanderploeg@zzmk.uzh.ch

Received 22 January 2009

Revised 4 March 2009

Accepted 7 March 2009

\author{
Jan R. van der Ploeg \\ Institute of Oral Biology, University of Zurich, Plattenstrasse 11, CH-8032 Zurich, Switzerland
}

\begin{abstract}
Clustered regularly interspaced short palindromic repeats (CRISPR) consist of highly conserved direct repeats interspersed with variable spacer sequences. They can protect bacteria against invasion by foreign DNA elements. The genome sequence of Streptococcus mutans strain UA159 contains two CRISPR loci, designated CRISPR1 and CRISPR2. The aims of this study were to analyse the organization of CRISPR in further $S$. mutans strains and to investigate the importance of CRISPR in acquired immunity to M102-like phages. The sequences of CRISPR1 and CRISPR2 arrays were determined for 29 S. mutans strains from different persons. More than half of the CRISPR 1 spacers and about $35 \%$ of the CRISPR2 spacers showed sequence similarity with the genome sequence of $\mathrm{M} 102$, a virulent siphophage specific for $S$. mutans. Although only a few spacers matched the phage sequence completely, most of the mismatches had no effect on the amino acid sequences of the phage-encoded proteins. The results suggest that $S$. mutans is often attacked by M102-like bacteriophages, and that its acquisition of novel phage-derived CRISPR sequences goes along with the presence of $S$. mutans phages in the environment. Analysis of CRISPR1 of M102-resistant mutants of S. mutans OMZ 381 showed that some of them had acquired novel spacers, and the sequences of all but one of these matched the phage M102 genome sequence. This suggests that the acquisition of the spacers contributed to the resistance against phage infection. However, since not all resistant mutants had new spacers, and since the removal of the CRISPR1 array in one of the mutants and in wild-type strains did not lead to loss of resistance to infection by $\mathrm{M} 102$, the acquisition of resistance must be based on further elements as well.
\end{abstract}

\section{INTRODUCTION}

The human oral cavity is inhabited by more than 500 different bacterial species (Paster et al., 2001), of which only a few cause disease. Dental caries, one of the most frequent diseases in humans, is caused by micro-organisms that metabolize dietary sugars into acids. The concomitant decrease in $\mathrm{pH}$ results in demineralization of the hard tissues of teeth. Mutans streptococci, in particular Streptococcus mutans, are considered the principal aetiological agents of dental caries. Most efforts to develop

Abbreviation: CRISPR, clustered regularly interspaced short palindromic repeats.

A supplementary figure and six supplementary tables are available with the online version of this paper. The supplementary figure shows a comparison of part of the cas 1 gene from Streptococcus mutans, Streptococcus equi subsp. zooepidemicus and Streptococcus pyogenes strains. The supplementary tables list oligonucleotides used in this study, CRISPR1 and CRISPR2 spacer sequences, sequences of the CRISPR1 and CRISPR2 repeats, and similarity of $S$. mutans CRISPR spacers to sequences other than those of M102. strategies for prevention of this disease are therefore aimed towards reduction of $S$. mutans by interference with colonization or by passive or active immunization while leaving beneficial micro-organisms untouched. Phage therapy, the application of bacteriophages to combat bacterial infections, has regained interest during the last few years due to the increase in bacterial resistance to conventional antibiotics. In principle, phage therapy could be used to specifically remove $S$. mutans from dental plaque.

Relatively little is known about $S$. mutans bacteriophages and about the role they play in the ecology of $S$. mutans in the oral cavity. It is difficult to isolate bacteriophages specific for mutans streptococci (Bachrach et al., 2003; J. R. van der Ploeg, unpublished results). Armau et al. (1988) isolated 16 lytic bacteriophages specific for strains of $S$. mutans and Streptococcus sobrinus by screening more than 1000 plaque samples. Three of these, phages M102, e1 and $\mathrm{f} 2$, appear to be similar, as suggested by comparable virion sizes, GC content, DNA-DNA hybridization and restric- 
tion fragment analysis, although the phages show different serotype specificity (Delisle \& Rostkowski, 1993). The genome sequence of M102, which is specific for S. mutans serotype c strains, has been determined (van der Ploeg, 2007). Another serotype c-specific bacteriophage, M101, appears to be closely related to M102 (van der Ploeg, 2007).

One of the weaknesses of phage therapy is the eventual development of resistance against bacteriophage infection. S. mutans strain OMZ 381 is one of only a few strains supporting propagation of M102 (Delisle \& Rostkowski, 1993; J. R. van der Ploeg, unpublished results). The causes of this limited host range are not known. Several mechanisms that render lactic acid bacteria resistant to phage infection have been described: inhibition of phage adsorption, inhibition of DNA injection, restriction modification of phage DNA, and abortive infection (Forde \& Fitzgerald, 1999). In the case of S. mutans, nothing is known about the last three mechanisms, but it has been shown that M102 does adsorb also to S. mutans serotype $\mathrm{c}$ strains that do not support propagation (Shibata et al., 2009). This suggests that factors different from phage adsorption must determine resistance of $S$. mutans serotype c strains to infection by M102.

Recently, a novel phage resistance mechanism, which relies on clustered regularly interspaced short palindromic repeats (CRISPR) and a set of CRISPR-associated genes (cas), has been discovered (Barrangou et al., 2007). CRISPR clusters consist of direct repeats of $24-47 \mathrm{bp}$, which are separated by unique spacers of 25-72 bp (Makarova et al., 2006; Sorek et al., 2008). Several CRISPR subtypes exist and each subtype has its own particular set of cas genes (Kunin et al., 2007). CRISPR are found in about $40 \%$ of sequenced bacterial genomes and in about $90 \%$ of sequenced archaeal genomes (Sorek et al., 2008). Frequently, a high number of the CRISPR spacer sequences show homology to bacteriophage or plasmid sequences. It has been shown that Streptococcus thermophilus mutants with acquired resistance to phage infection have incorporated an additional CRISPR spacer, whose sequence matches $100 \%$ with part of the phage genome (Barrangou et al., 2007). Removal of this spacer sequence leads to sensitivity to phage infection (Barrangou et al., 2007). Apart from providing resistance to bacteriophages, CRISPR interference has also been shown to block plasmid DNA conjugation and transformation (Marraffini \& Sontheimer, 2008).

In the present study, the incidence and the composition of CRISPR in $S$. mutans was examined and the role of CRISPR in protection against bacteriophage infection was investigated.

\section{METHODS}

Strains, plasmids and phages, and growth conditions. S. mutans strains used are listed in Table 1. Unless specified otherwise, they were routinely grown at $37{ }^{\circ} \mathrm{C}$ under anaerobic conditions or in an atmosphere of $10 \% \mathrm{CO}_{2}$ and $90 \%$ air in THY medium, which consists of Todd-Hewitt medium (Beckton-Dickinson) with $0.3 \%$ yeast extract (Oxoid). Solid medium contained $1.5 \%$ agar. Bacteriophages M102 and M101 were propagated in S. mutans strain OMZ 381 as described previously (van der Ploeg, 2007). When required, erythromycin was added to a final concentration of $10 \mu \mathrm{g} \mathrm{ml}^{-1}$. Escherichia coli JM109 was used as the host for propagation of plasmids and was grown in LB medium at $37{ }^{\circ} \mathrm{C}$ with aeration. When required, erythromycin was added to a final concentration of $200 \mu \mathrm{g} \mathrm{ml}^{-1}$.

PCR amplification and DNA sequencing. Chromosomal DNA was isolated with a GenElute bacterial genomic DNA kit (Sigma). CRISPR1 arrays were amplified by PCR with primers CRu and CRd or Cru2 and Crd2 (Supplementary Table S1). For amplification of CRISPR2 arrays, primers Cau and Cad or Cau2 and Cad2 were used. Taq DNA polymerase (New England Biolabs) or Jumpstart Taq polymerase (Sigma) were employed. The PCR program consisted of an initial denaturation for $3 \mathrm{~min}$ at $95{ }^{\circ} \mathrm{C}$, followed by 30 cycles of annealing for $30 \mathrm{~s}$ at $55{ }^{\circ} \mathrm{C}$, extension for $3 \mathrm{~min}$ at $72{ }^{\circ} \mathrm{C}$ and denaturation for $30 \mathrm{~s}$ at $95{ }^{\circ} \mathrm{C}$. PCR products were purified with a Jetquick PCR purification kit (Genomed) and directly sequenced using dye-terminator technology (Microsynth) with one of the primers used for amplification. When required, a primer-walking strategy was employed for the sequencing of large CRISPR arrays.

The serotypes of S. mutans strains OMZ 380, OMZ 382, OMZ 737 and OM1079 were determined by PCR analysis (Shibata et al., 2003). In addition, spaP, atlA, SMU.1757c and SMU.1762c from some strains were amplified by PCR with the primers listed in Supplementary Table S1 and sequenced with one of the primers used for amplification. Specific fragments of bacteriophage M101 were amplified and sequenced using primers specified in Supplementary Table S1.

Bioinformatic analyses. CRISPR spacers were identified by using the CRISPR finder tool (available at http://crispr.u-psud.fr/; Grissa et al., 2007) combined with visual inspection of the sequence. Similarity of spacers to M102 was analysed by TBLASTX (using 0.1 as e-value cut off). Similarity between spacer and database sequences was searched for by BLAST analysis. WebLogo (Crooks et al., 2004) was used to visualize sequence consensus motifs.

Isolation of mutants resistant to infection by phage M102. S. mutans strain OMZ 381 is one of a few strains which support propagation of bacteriophage M102 (Delisle \& Rostkowski, 1993; Shibata et al., 2009). OMZ 381 was therefore chosen for isolation of phage-resistant mutants. For this, $100 \mu \mathrm{l}$ of serial dilutions of an exponentially growing OMZ 381 culture was mixed with $\sim 3 \times 10^{8}$ p.f.u. of phage M102. After incubation at $37{ }^{\circ} \mathrm{C}$ for $30 \mathrm{~min}, 4 \mathrm{ml}$ M1D soft agar (van der Ploeg, 2007) was added, immediately poured on an M1D plate and incubated for $48 \mathrm{~h}$ at $37^{\circ} \mathrm{C}$. Colonies that appeared were recloned on M1D agar and tested for resistance to phage infection.

One further M102-resistant mutant was isolated in the course of an animal experiment. In brief, in a study approved by the 'Veterinäramt des Kantons Zürich' and conformed to the Swiss laws on animal protection, 10 specific-pathogen-free, caries-susceptible OsborneMendel rats (Institute of Oral Biology, University of Zurich, Zurich, Switzerland) were associated orally with S. mutans OMZ 381 as described elsewhere (Thurnheer et al., 2008). Thereafter, phage M102 $\left(10^{5}\right.$ p.f.u., $0.1 \mathrm{ml}$ ) was applied in the oral cavity of the rats (twice daily on days $23,24,25$ and 26). Oral swabs were taken from all rats on days 29,34 and 43 and suspended in $0.9 \% \mathrm{NaCl}$, and various dilutions were plated on $S$. mutans selective medium. Three $S$. mutans colonies from each rat were tested for resistance to phage M102. Only two mutants were obtained, both from day 43, of which one, OMZ 432, was used for further investigation. 
Table 1. Strains of $S$. mutans used in this study

\begin{tabular}{|c|c|c|c|c|c|c|}
\hline Strain & $\begin{array}{c}\text { Original } \\
\text { designation }\end{array}$ & Serotype & $\begin{array}{l}\text { Presence of } \\
\text { CRISPR1 }\end{array}$ & $\begin{array}{l}\text { Presence of } \\
\text { CRISPR2 }\end{array}$ & Origin & Reference or source \\
\hline OMZ 7 & NCTC $10449 \dagger$ & c & 3 & 7 & UK & Edwardsson (1968) \\
\hline OMZ 20 & MT118 & c & 33 & $-\ddagger$ & Japan & Hamada \& Ooshima (1975) \\
\hline OMZ 25 & MT142 & c & 18 & - & Japan & Hamada \& Ooshima (1975) \\
\hline OMZ 26 & C67-1 & c & - & 6 & The Netherlands & de Stoppelaar et al. (1971) \\
\hline OMZ 27 & C67-25 & c & - & 50 & The Netherlands & de Stoppelaar et al. (1971) \\
\hline OMZ 30 & MT12 & c & - & 42 & Japan & Hamada \& Ooshima (1975) \\
\hline OMZ 64 & IB1600 & c & 12 & - & Sweden & Krasse (1966) \\
\hline OMZ 67 & GS-5 & c & 22 & 2 & USA & Gibbons et al. (1966) \\
\hline OMZ 70 & ATCC $33535 \S$ & c & - & - & Switzerland & Guggenheim (1968) \\
\hline OMZ 186 & $\mathrm{KPSK} 2=\mathrm{JC} 2$ & c & 5 & - & Sweden & Carlsson (1967) \\
\hline OMZ 380 & P34 & c & 8 & - & France & $\begin{array}{l}\text { G. Tiraby, Université Paul Sabatier, } \\
\text { Toulouse, France }\end{array}$ \\
\hline OMZ 381 & $\mathrm{P} 42$ & c & 6 & - & France & G. Tiraby \\
\hline OMZ 382 & S61 & c & 31 & - & France & G. Tiraby \\
\hline OMZ 737 & R9 & c & - & - & UK & D. J. Bradshaw, CAMR, Salisbury, UK \\
\hline OMZ 918 & UA159 & c & 6 & 1 & USA & ATCC \\
\hline OMZ 949 & 'NG8' & c & 5 & - & & D. Cvitkovitch, University of Toronto \\
\hline OMZ 960 & UA130 & c & - & 13 & USA & G. Spatafora, Middlebury College \\
\hline OMZ 961 & 'NG8' & c & 5 & - & & G. Spatafora, Middlebury College \\
\hline OMZ 1040 & PK1 & c & 20 & - & USA & Gibbons et al. (1966) \\
\hline OMZ 1079 & & c & 38 & - & Switzerland & J. R. van der Ploeg, unpublished results \\
\hline At10 & & c & 6 & - & Sweden & Edwardsson (1968) \\
\hline L18 & & c & 15 & - & Finland & Grönroos \& Alaluusua (2000) \\
\hline LML5 & & c & - & - & UK & Waterhouse \& Russell (2006) \\
\hline LML7 & & c & 9 & - & UK & Waterhouse \& Russell (2006) \\
\hline MT4863 & & c & 6 & - & Japan & Hamada et al. (1980) \\
\hline OMZ 125 & LM7 & e & 26 & - & USA & Gibbons et al. (1966) \\
\hline MT4653 & & e & 16 & - & Japan & Hamada et al. (1980) \\
\hline OMZ 175 & & $\mathrm{f}$ & - & 1 & Switzerland & Guggenheim (1968) \\
\hline OM98x & & $\mathrm{k}$ & 25 & 33 & Japan & T. Sato, Tokyo Dental College, Chiba, Japan \\
\hline
\end{tabular}

${ }^{*}$ The number of spacers is shown.

$\dagger$ NCTC, National Collection of Type Cultures.

$\$-$, None detected.

§ATCC, American Type Culture Collection.

Construction of mutations in CRISPR1 and SMU.1405c. For construction of mutants with a deletion in CRISPR1, a doublecrossover replacement strategy was employed. The region $5^{\prime}$ to CRISPR1 was amplified by PCR with primers CR1 and CR2, using genomic DNA from S. mutans OMZ 1001 as template (OMZ 1001 is a derivative of UA159 with a high transformation frequency). The product was digested with BamHI and HindIII and cloned into plasmid pFW15 (Podbielski et al., 1996) to create plasmid pOMZ357. Subsequently, the region $3^{\prime}$ to CRISPR1 was amplified with primers CR3 and CR4, digested with EcoRI and NcoI, and cloned into pOMZ357 to create pOMZ358. Plasmid pOMZ358 was linearized by digestion with BamHI and introduced into S. mutans strains by transformation of naturally competent cells (Perry \& Kuramitsu, 1981). Selection for chromosomal integration was done on THY agar plates containing erythromycin. Correct integration was confirmed by PCR analysis.

A similar strategy was used for the construction of SMU.1405c knockout mutants. An approximately $500 \mathrm{bp}$ fragment internal to SMU.1405c was amplified by PCR with primers $1405 \mathrm{c} 1$ and $1405 \mathrm{c} 2$. The fragment was digested with BamHI and HindIII and cloned into
pFW15 to give plasmid pOMZ417. A second fragment harbouring the $3^{\prime}$ end of SMU.1405c was amplified with primers 1405c3 and 1405c4, digested with PstI and EcoRI, and cloned into pOMZ417 to yield plasmid pOMZ418. The EcoRI-linearized plasmid pOMZ418 was introduced into different strains of $S$. mutans by transformation. Selection for and confirmation of plasmid integration were done as described above.

\section{RESULTS}

\section{Bioinformatic analysis of CRISPR arrays and cas genes in S. mutans UA159}

The annotated genome of S. mutans UA159 (Ajdić et al., 2002) was found to harbour two CRISPR loci, designated CRISPR1 and CRISPR2. Two S. mutans CRISPR types have also been identified recently by others (Haft et al., 2005; Horvath et al., 2008a). As outlined in Fig. 1(a), CRISPR1 is 

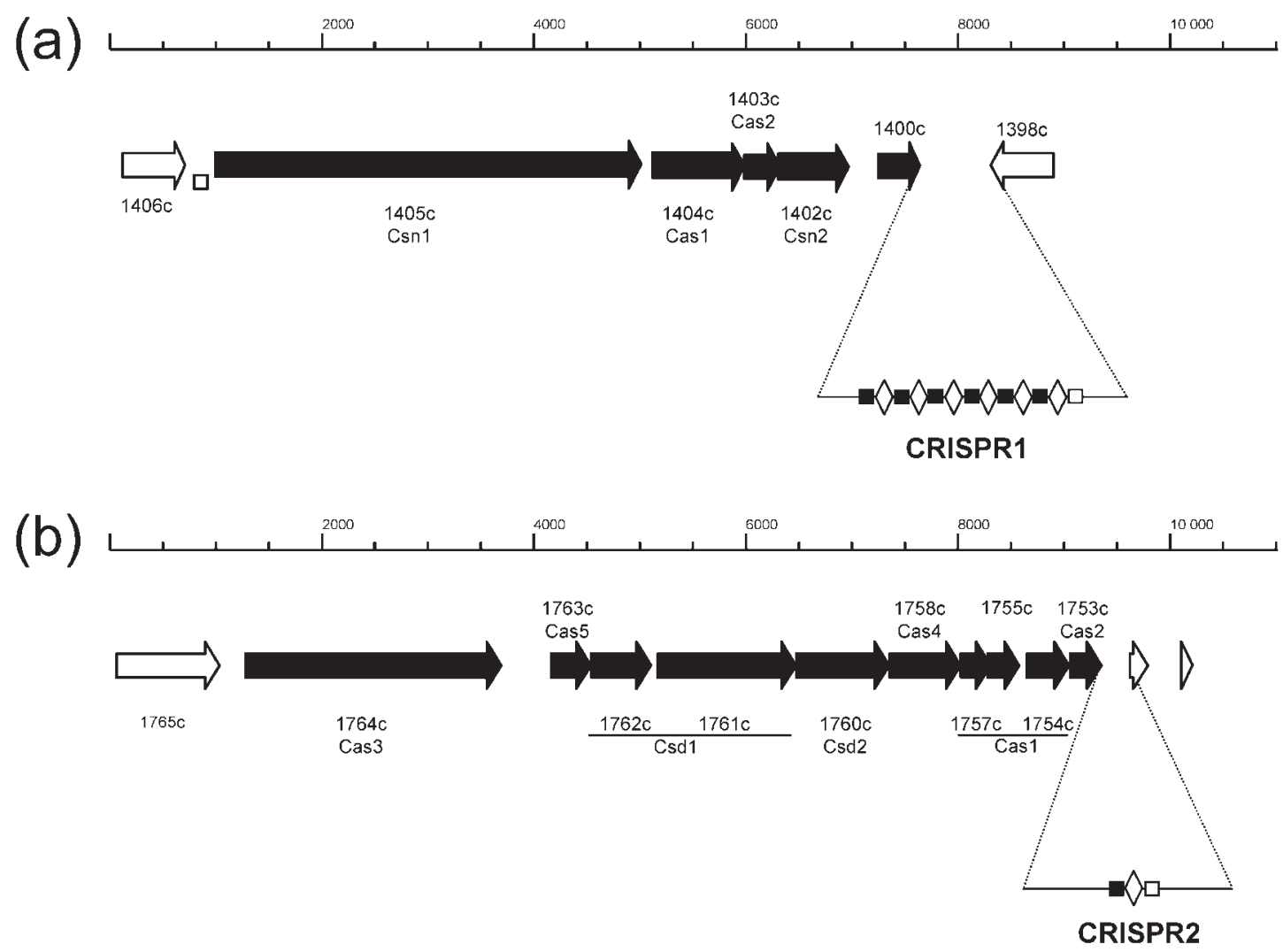

Fig. 1. Organization of CRISPR1 (a) and CRISPR2 (b) arrays and associated genes in S. mutans UA159. Lines below the ORF numbers show the size that the gene would have if the frame shift or stop codons (see text) were removed. CRISPR repeats are indicated by boxes (black, conserved; white, degenerated). Spacers are indicated by diamonds.

located between ORFs SMU.1400 and SMU.1398, and contains six identical copies of a partially palindromic sequence of $36 \mathrm{bp}$ (5'-GTTTTAGAGCTGTGTTGTTTCGAATGGTTCCAAAAC-3'). A seventh degenerated repeat with $67 \%$ identity (5'-GTTTTAGAGCCATGTTAGTTACTGATTTACTAAAAT-3') is located proximal to SMU.1398. All repeats are interspersed by spacers of $30 \mathrm{bp}$ in size. The CRISPR1 array is preceded by four ORFs (SMU.1405c, SMU.1404c, SMU.1403c and SMU.1402c), whose amino acid sequences show similarity to those of cas genes of the Nmeni subtype (Haft et al., 2005). This subtype is usually associated with the CRISPR repeat cluster 10 (Kunin et al., 2007), to which the S. mutans CRISPR1 repeat also belongs. An additional partially homologous repeat (5' -TTTAACTTGCTGTGTTGTTTCGAATGATTCCAACAC-3') is present upstream of SMU.1405c (Fig. 1), and is a common feature of this CAS/CRISPR subtype (Haft et al., 2005). Another annotated ORF, SMU.1399, overlaps with the CRISPR1 array (not shown). Its deduced amino acid sequence shows no similarity to proteins of known function.

The CRISPR2 locus of UA159 is located between SMU.1753c and SMU.1752c, and consists of two 32 bp partially homologous repeats (5'-GTCGCACCCTTCACGGGTGCGTGGATTGAAAT- $3^{\prime}$ and $5^{\prime}$-GTCGCACCC-
TTTAAAGGTTGGGTTTGCTTTTT-3'), separated by a 34 bp spacer (Fig. 1b). The first repeat is identical to a CRISPR repeat present in the genome of Streptococcus pyogenes M49 (McShan et al., 2008). It is a member of repeat cluster 3 (Kunin et al., 2007), associated with subtype Dvulg cas genes (Haft et al., 2005). The genes from SMU.1764c to SMU.1753c located upstream of the first repeat (Fig. 1) belong to this subtype. However, two of these genes appear to be truncated by a frame shift mutation ( $c s d 1$ and cas 1 ) and one by a premature stop codon (cas1).

\section{Distribution of CRISPR1 and CRISPR2 in mutans streptococci}

The distribution of CRISPR1 and CRISPR2 in 29 isolates of S. mutans (Table 1) was investigated by PCR analysis and sequencing, using primers derived from the sequences bordering the CRISPR of $S$. mutans UA159. Since it turned out that strains OMZ 186, OMZ 949 and OMZ 961 contained identical CRISPR (see below), OMZ 949 and OMZ 961 were omitted from the analysis. CRISPR1 was detected more frequently (in 19 out of 27 strains) than CRISPR2 (nine out of 27). Both CRISPR clusters were 
present in only four strains (OMZ 7, UA159, OMZ 67 and OM98x). Three serotype $\mathrm{c}$ strains yielded neither a CRISPR1 nor a CRISPR2 PCR product. Most positive strains displayed many more repeats and spacers in both CRISPR1 and CRISPR2 than S. mutans UA159 (Fig. 2a, b).

A total of 305 CRISPR1 spacers were found in the 19 strains that yielded a PCR product; the mean number was 16.0 (range 3-38; Supplementary Table S2). Within CRISPR2, 155 spacers were found in nine positive strains, with a mean number of 17.2 spacers per strain (range 1-50).

\section{Sequence analysis of CRISPR1 and CRISPR2 spacers and repeats}

Spacers. More than $90 \%$ of the CRISPR1 spacer sequences were $30 \mathrm{bp}$ in size; the remaining spacers consisted of 28, 29 or 31 bp (Supplementary Table S2). Most were unique, and only the terminal spacer (preceding the terminal degenerated repeat) showed some conservation. A total of six different terminal spacers were found, of which one occurred in eight different strains. Apart from the conservation of the last spacer, there were only eight identical CRISPR1 spacer pairs, and these pairs were always located close to each other within a single strain. CRISPR1 from OM98x was unusual, with a block of three spacers in repeat. These duplications may have been selected in response to phage predation, as they could lead to an increased efficiency of inhibition of the target nucleic acid. Alternatively, identical spacers could have been generated by errors of the CRISPR machinery.

With respect to CRISPR2 spacers, sequences of $34 \mathrm{bp}$ occurred most frequently, followed by 35,33 and $36 \mathrm{bp}$ (Supplementary Table S3). As for CRISPR1, identical spacers were detected only occasionally. An exception was again the terminal spacer sequence, which occurred in three variants.

Repeats. The sequences of the CRISPR1 and CRISPR2 repeats are summarized in Supplementary Tables S4 and S5, respectively. In contrast to spacer diversity, no sequence variation was found in the regular CRISPR1 repeats, except for strain MT4863, where the last residue was a T instead of C. The terminal CRISPR1 repeat was found in three variants. Regular CRISPR2 repeats occurred in two variants, with only one base pair being affected. The terminal CRISPR2 repeat was different from the regular CRISPR2 repeats and also occurred in two variants.

\section{Inactive CRISPR2 in UA159 due to mutations}

As described above, cas 1 and $c s d 1$, which are associated with CRISPR2, appeared to be truncated in UA159. Since this strain contains only one CRISPR 2 spacer, it has been postulated that the mutations in casl and csd 1 render the CRISPR2 system inactive (Horvath et al., 2008a). To find evidence for this hypothesis, part of the cas1 gene (861 bp) from strains OMZ 960 and OM98x was sequenced. The data show that these strains contain 13 and 33 CRISPR2 spacers respectively, which suggests an active CRISPR2 system. Compared with the sequence of UA159, two base pairs (GT) are inserted into both OMZ 960 and OM98x (Supplementary Figure S1). In addition, the stop codon present in the UA159 sequence is absent in OMZ 960 and OM98x.

Likewise, part of the sequence of $c s d 1$ (660 bp) from OMZ 960 was determined. Comparison with UA159 revealed two differences: an A-G transition, which has no influence on the amino acid sequence of the encoded protein, and an insertion of $1 \mathrm{bp}$ within a homopolymeric dA tract in the UA159 sequence, which results in a frame shift (data not shown). Together, these results suggest that both cas1 and csd1 are non-functional in UA159, implying that UA159 has lost the ability to incorporate novel CRISPR2 spacers.

\section{Identical CRISPR1 sequences in OMZ 186 and OMZ 961}

The CRISPR1 sequences of OMZ 961 (NG8, obtained from G. Spatafora, Middlebury College) and OMZ 186 (KSPK2 or JC-2) were identical. This appeared rather unlikely, since the strains had been isolated in New Guinea and Sweden, respectively (R. Russell, personal communication). Hence, the CRISPR1 sequence of OMZ 949 (NG8, obtained from D. Cvitkovitch, University of Toronto) was determined as well and was found to be identical to that of CRISPR1 from OMZ 961 and OMZ 186. To investigate this further, a part of the sequence of the spaP gene of these three strains was determined, since it had been reported that spaP is different in KSKP2 and NG8 (Demuth et al., 1990; P. Crowley, personal communication). However, in the present study, the spaP sequences obtained from OMZ 961 and OMZ 186 were identical to KSPK2 (data not shown), which strongly suggests that some laboratories hold the same strain under different names.

\section{Similarity of CRISPR spacers to bacteriophage M102}

Of the 305 CRISPR1 spacer sequences determined, only five matched the bacteriophage M102 genome sequence exactly. However, an additional 172 CRISPR1 spacers showed sequence similarity to M102 (more than 17 identical residues; Fig. 2a). The majority of them contained mismatches at degenerate positions, and the encoded amino acid sequence remained unchanged compared with M102. Of 201 mismatches investigated, 161 concerned the third position of the codon of the targeted M102 ORF, and were mostly silent ( $n=152$ ). The remaining 49 mismatches resulted in 30 amino acid changes.

Of the 155 CRISPR2 spacers, 55 (35\%) showed sequence similarity to M102 (more than 22 identical residues; Fig. $2 \mathrm{~b}$ ), but none matched perfectly. Again, the majority of the mismatches were positioned at the third base of the codon (69 out of 93 mismatches investigated). In total, only 18 amino acid changes resulted from these mismatches. 
(a)

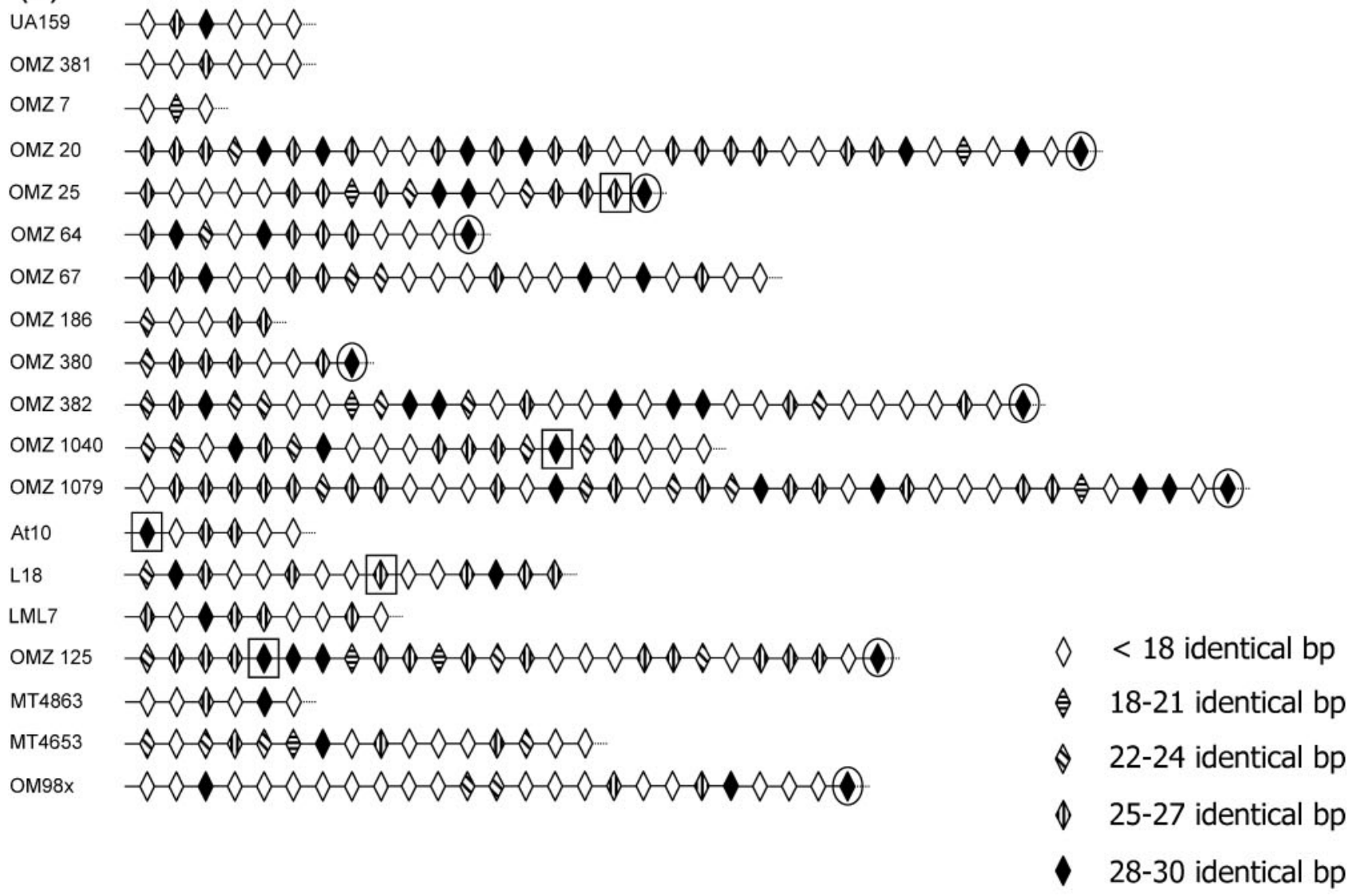

(b)

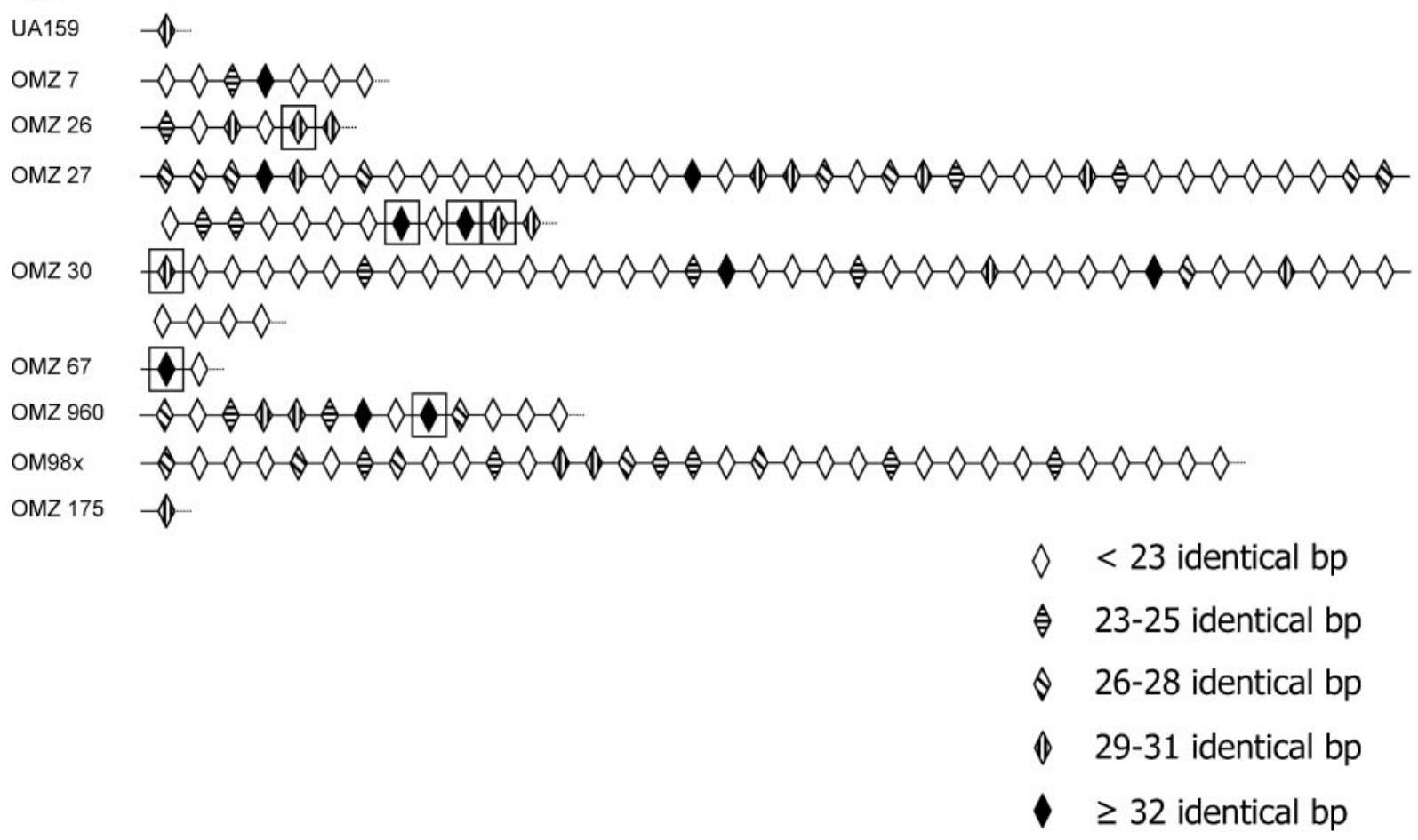

$\diamond<23$ identical bp

- 23-25 identical bp

- 26-28 identical bp

1) 29-31 identical bp

$\checkmark \geq 32$ identical bp 
Fig. 2. Analysis of CRISPR1 (a) and CRISPR2 (b) in S. mutans. Repeats are shown as lines; the last non-conserved repeat is shown as a dotted line. Spacers are represented by diamonds; shading indicates similarity to known sequences. Spacers that are encircled have identical sequences; spacers that are surrounded by a rectangle show similarity to sequences different from the genome of phage M102.

It has been shown that within a CRISPR repeat family, bacteriophage-derived sequences located adjacent to the proto-spacers, the region in the phage genome correspoding with the spacer, exhibit conservation (Bolotin et al., 2005; Deveau et al., 2008; Horvath et al., 2008b). In this study, comparison of the sequences adjacent to CRISPR1 proto-spacers showed conservation within the three-base stretch at the $3^{\prime}$ end $\left(5^{\prime}\right.$-WRR; Fig. 3a). Two submotifs were recognized in different strains. In OMZ 64, OMZ 382, OMZ 1040, OMZ 1079, OMZ 381 and MT4863, a 5'WAAR-3' motif is present (Fig. 3b), whereas the remaining strains show a $5^{\prime}$-WGG-3' motif (Fig. 3c). In contrast, no conservation was observed next to the $5^{\prime}$ end of the protospacers (results not shown).

With regard to CRISPR2, the sequences $3^{\prime}$ to the CRISPR2 proto-spacers showed no conservation, whereas the three bases located at the $5^{\prime}$ site commonly exhibited the motif $5^{\prime}$-TTC-3' (Fig. 3d).

\section{Similarity of CRISPR spacers to other sequences}

Apart from the evident sequence similarity to bacteriophage M102, some CRISPR1 and CRISPR2 spacers showed similarity to other invading mobile elements, namely bacteriophages from other streptococci, plasmids and transposons (Supplementary Table S6). Three CRISPR1 and CRISPR2 spacer sequences showed similarity to a $48 \mathrm{~kb}$ plasmid present in three copies in the genome of Streptococcus agalactiae pNEM316-1 (Glaser et al., 2002). Some CRISPR2 spacers from OMZ 26, OMZ 27 and OMZ 960 match the sequence of the genomic island Tnsmu1, which encompasses SMU.191c to SMU.221c and is present in UA159 and other S. mutans strains (Waterhouse et al., 2007). The clustering of spacers in OMZ 27 hints at repeated attacks of Tn1smu or at simultaneous incorporation of multiple spacers from the Tn1smu genome. Two CRISPR1 spacers matched the ORFs SMU.1705 and SMU.820 of S. mutans UA159.

(a)

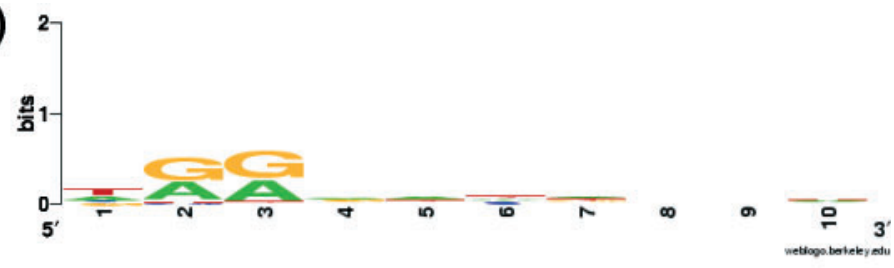

(b)

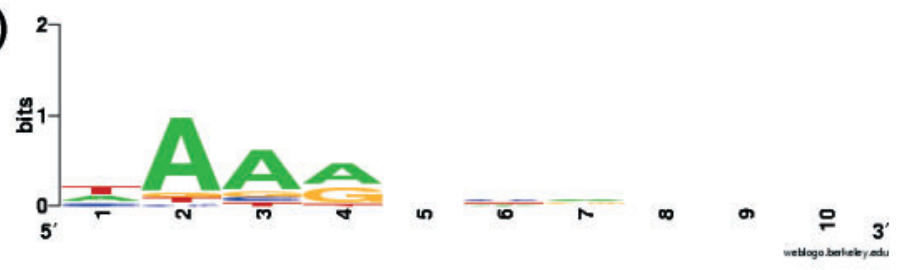

(c)

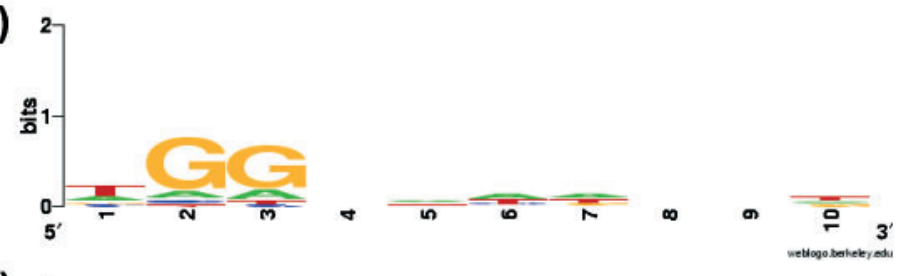

(d)

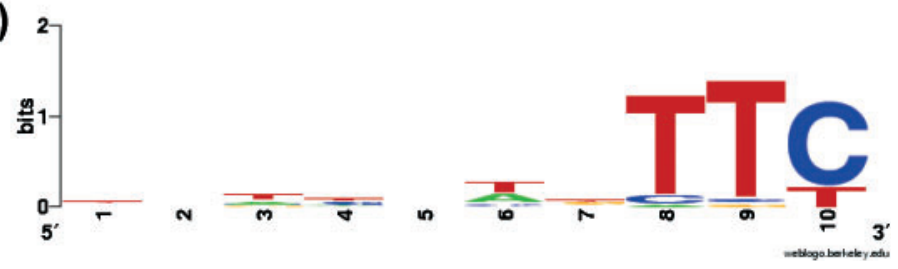

Fig. 3. WebLogo motifs of sequences $5^{\prime}$ and $3^{\prime}$ to proto-spacers. (a) Sequences located 3' to CRISPR1 proto-spacers; (b) sequences $3^{\prime}$ to CRISPR1 proto-spacers targeted by spacers from strains OMZ 64, OMZ 382, OMZ 1040, OMZ 1079 and OMZ 381; (c) sequences $3^{\prime}$ to CRISPR1 proto-spacers targeted by spacers from strains OMZ 20, OMZ 25, OMZ 67, OMZ 186, OMZ 380, OMZ 382, UA159, At10, L18, LML7, MT4863, OMZ 125, Mt4653 and OM98x; (d) sequences $5^{\prime}$ to CRISPR2 proto-spacers. 


\section{Analysis of CRISPR1 in M102-resistant mutants}

Spontaneous mutants of OMZ 381 resistant to infection by M102 could easily be isolated. PCR analysis showed that in 13 of 57 analysed mutants the size of the CRISPR array had increased by approximately the size of one spacer and one repeat (Table 2). The acquisition of a novel spacer and repeat in these mutants was confirmed by DNA sequencing. In OMZ 432, a strain isolated from a rat whose oral cavity had been inoculated with the wild-type strain (OMZ 381 ) and then challenged with phage M102, two additional CRISPR1 spacers were detected (Table 2).

With one exception (OMZ 381-M5) all newly acquired spacers and repeats were inserted proximal to the CRISPR1 leader. This corroborates results from earlier studies with phage-resistant S. thermophilus mutants (Barrangou et al., 2007; Deveau et al., 2008). In all but one case (OMZ 381M71), the novel spacer sequences matched the genome sequence of M102 perfectly. In OMZ 381-M71, one mismatch at the ultimate $5^{\prime}$ base of the proto-spacer is present. In some mutants, nearly identical spacers are incorporated (Table 2).

In mutants M3, M60 and M71, the spacer matched the same phage sequence, but the length of the spacer differed. This was also found for two other mutants (OMZ 432 and M56). The region 3' to the proto-spacer sequence exhibited a conserved motif (YAAAWY) that is similar to the CRISPR1 motif described above (Fig. 3b).

Table 2. M102-resistant mutants of OMZ 381

\begin{tabular}{|c|c|c|c|c|c|c|c|}
\hline Mutant & Novel spacer sequence & $\begin{array}{l}\text { Size } \\
(\text { bp) }\end{array}$ & $\begin{array}{c}\text { M102 } \\
\text { co-ordinates }^{*}\end{array}$ & $5^{\prime}$ Sequence $\dagger$ & $3^{\prime}$ Sequence $\neq$ & $\begin{array}{l}\text { Sensitivi- } \\
\text { ty to } \\
\text { M101\$ }\end{array}$ & M101 proto-spacer|| \\
\hline OMZ432 & $\begin{array}{l}\text { AAGATGGCTGAAGAG- } \\
\text { ACCGGGGTTAACGCC }\end{array}$ & 30 & $12228-12198$ (r) & TACCATTTCC & TAAATTAGCA & - & $\begin{array}{l}\text { AAGATGGCTGAAGAG- } \\
\text { ACCGGGGTTAACGCC }\end{array}$ \\
\hline $\begin{array}{l}\mathrm{OMZ} \\
381-\mathrm{M} 3\end{array}$ & $\begin{array}{l}\text { ATATGTAATAGGTCA- } \\
\text { TAATGATGATGGCAC }\end{array}$ & 30 & $17268-17297$ & TTGCCCGTGA & TAAAACAATT & - & $\begin{array}{l}\text { ATATTGTĀATAGGTCATA- } \\
\text { ATGATGATGGCAC }\end{array}$ \\
\hline $\begin{array}{l}\text { OMZ } \\
\text { 381-M5 }\end{array}$ & $\begin{array}{l}\text { GCAGTCAGTAGCGCA- } \\
\text { ACAAGAGCTACAGGC }\end{array}$ & 30 & $16560-16579$ & TTGCAGACGC & TAAAGCAAGT & - & $\begin{array}{l}\underline{\text { ACAATCAGTCGCACAAC- }} \\
\text { AAGAACTGCAAGC }\end{array}$ \\
\hline $\begin{array}{l}\text { OMZ } \\
\text { 381-M19 }\end{array}$ & $\begin{array}{l}\text { TATGCGTTGGATTGTT- } \\
\text { TTTGCTCTGTCTA }\end{array}$ & 29 & $21869-21841(\mathrm{r})$ & TAGCACCGAT & TAAAGCCTTC & - & $\begin{array}{l}\text { TGTGCGTTGAATTGTC- } \\
\text { TTTGCTCTGTCTA }\end{array}$ \\
\hline $\begin{array}{l}\text { OMZ } \\
\text { 381-M54 }\end{array}$ & $\begin{array}{l}\text { GATAATGCTTTTGCCA- } \\
\text { AAGCACAGCTAATC }\end{array}$ & 30 & $4621-4650$ & CGACGTTATC & CAAAACGCTT & - & $\begin{array}{l}\text { GATAATGTTTTTGCTAA- } \\
\text { AGCGCAGITAATC }\end{array}$ \\
\hline $\begin{array}{l}\text { OMZ } \\
\text { 381-M56 }\end{array}$ & $\begin{array}{c}\text { CAAGATGGCTGAAGAG- } \\
\text { ACCGGGGTTAACGCC }\end{array}$ & 31 & $12228-12198$ (r) & TTACСАТTTC & TAAATTAGCT & - & $\begin{array}{l}\text { CAAGATGGCTGAAGAGA- } \\
\text { CCGGGGTTAACGCC }\end{array}$ \\
\hline $\begin{array}{l}\mathrm{OMZ} \\
381-\mathrm{M} 60\end{array}$ & $\begin{array}{l}\text { AATATGTAATAGGTCAT- } \\
\text { AATGATGATGGCAC }\end{array}$ & 31 & $17267-17297$ & TTTGCCCGTG & TAAAACAATT & + & $\begin{array}{l}\text { AATATGTAATAGGTCAT- } \\
\text { AATGATGATGGCAC }\end{array}$ \\
\hline $\begin{array}{l}\text { OMZ } \\
\text { 381-M62 }\end{array}$ & $\begin{array}{l}\text { TAAGAATTTGTTTTC- } \\
\text { TTTGGCATTCAGCCA }\end{array}$ & 30 & 1993-1964 (r) & СТTТСААТТС & CAAATTCAAG & - & $\begin{array}{l}\text { TAAGAATTTGTTTTCTT- } \\
\text { TGGCATTCAGCCA }\end{array}$ \\
\hline $\begin{array}{l}\text { OMZ } \\
\text { 381-M63 }\end{array}$ & $\begin{array}{l}\text { GTGGCAAAAGTGATA- } \\
\text { GCTAGGAACATGCCT }\end{array}$ & 30 & $9798-9827$ & AATCGAAGAT & CAAATCATCA & - & $\mathrm{ND}$ \\
\hline
\end{tabular}

${ }^{\star}$ Co-ordinates of M102 proto-spacer; (r), reverse strand.

$\dagger$ Sequence $5^{\prime}$ to the M102 proto-spacer.

$¥$ Sequence $3^{\prime}$ to the M102 proto-spacer.

$\$+$, Sensitive; - , resistant to infection by phage M101.

IIProto-spacer sequence of M101. Underlined residues are different from the corresponding proto-spacer of M102.

$\mathbf{S}_{\mathrm{ND}}$, Not determined. 
Bacteriophage M101 is related to phage M102 (van der Ploeg, 2007). It was therefore investigated whether the M102-resistant mutants were also resistant to phage M101. The results showed that only mutants M60 and M71 were sensitive to M101 infection (Table 2). In these mutants, the acquired CRISPR1 spacer is homologous to a sequence of M102. However, this sequence is also present in the CRISPR1 spacer in mutant M3, which was resistant to infection by M101. To determine whether the differences in sensitivity to phage infection were related to differences in the proto-spacers, their sequences were determined. Some of the proto-spacer sequences in M101 were identical to those in M102 (M3, M60 and M62), whereas others were different. In fact, the proto-spacer in M101 differed from the novel spacer in mutant M5 by as many as seven nucleotides. Although M5 is resistant to infection by M101, it is hard to conceive that the acquired spacer in this mutant could provide protection. It has been reported that mutation of only one nucleotide in the phage proto-spacer rendered S. thermophilus bacteriophage 2972 unaffected by CRISPR-mediated resistance (Deveau et al., 2008).

\section{Inactivation of SMU.1405c or CRISPR1 is insufficient to render sensitivity to infection by the bacteriophage}

In order to confirm that resistance to phage M102 is conferred by the CRISPR1 system, mutants in the cas 5 gene SMU.1405c were constructed in OMZ 67, OMZ 961, OMZ 1001, OMZ 381-M3 and OMZ 381-M46. However, all resulting mutants remained resistant to infection by phage M102 (data not shown). Next, the CRISPR1 clusters of OMZ 7, OMZ 67, OMZ 1001, OMZ 381-M3 and OMZ 381-HS were deleted and replaced by an erythromycinresistance cassette. These mutants also remained resistant to infection by phage M102 (not shown).

\section{DISCUSSION}

The results of this study show that mutants of strain OMZ 381 which have become resistant to bacteriophage M102 have acquired novel CRISPR1 spacers that are most likely derived from the phage genomic DNA, since their sequences matched the genome sequence of M102. This finding is in agreement with studies of phage-resistant mutants of S. thermophilus (Barrangou et al., 2007; Horvath et al., 2008b). However, just as remarkable is the fact that in the majority of the M102-resistant OMZ 381 mutants no change in the CRISPR1 cluster had occurred. Thus, it is evident that in $S$. mutans there must exist other CRISPR1/2-independent and as yet unidentified mechanism(s) of acquired immunity to phage infection. In line with this conclusion are two further observations: first, inactivation of the CRISPR1 cluster in several different $S$. mutans strains and in a phage-resistant mutant of OMZ 381 with an acquired supplementary CRISPR1 spacer did not re-establish sensitivity to M102 infection; second, some of the novel M102-resistant mutants were also resistant to infection by M101, although there was only partial homology between the novel spacer sequence and the proto-spacer of M101. One possible explanation is that many S. mutans strains (other than UA159) may carry further, still unknown CRISPR cluster(s) with CRISPRassociated genes. In fact, some S. thermophilus strains possess three different CRISPR arrays (Horvath et al., 2008b). Whole genomic sequencing of other S. mutans strains may reveal whether one or more additional CRISPR clusters exist.

This study demonstrates that the prevalence, the composition and the sequences of CRISPR1 and CRISPR2 in $S$. mutans are highly variable. The polymorphic nature of CRISPR makes the two clusters useful markers for epidemiological studies. CRISPR1 was found to occur more frequently than CRISPR2. Although the choice of primers and or PCR conditions might have had an influence on the results, the distribution frequency was congruent with results from a comparative genomic hybridization study (Waterhouse et al., 2007). In that study, it was shown that a complete set of CRISPR1-associated genes (SMU.1405 to SMU.1400) is present in seven of nine strains investigated. Of these nine strains, seven were also part of the present work (GS5, At10, L18, LML5, LML7, MT4863 and MT4653), and in all but LML5, a CRISPR1 array was found. LML5 has been reported to lack CRISPR1-associated genes (Waterhouse et al., 2007), and indeed, no CRISPR1 array could be detected. With respect to CRISPR2, however, a complete set of CRISPR2-associated genes (SMU.1764 to SMU.1753) and a CRISPR2 array were present in a single strain only, GS5 (OMZ 67). These results agree with earlier observations that particular CRISPR repeat clusters are functionally coupled with particular CAS subtypes (Horvath et al., 2008b; Kunin et al., 2007).

My comparison of the sequences of cas 1 and $c s d 1$ from strain UA159 with those from other strains and species suggests that both genes are non-functional in UA159. It has been speculated that Cas1 functions to integrate novel spacers into CRISPR2 (Makarova et al., 2006). Hence, UA159 should have lost the ability to do so, and this would fit with the observation described here that UA159 contains only a single CRISPR2 spacer. This hypothesis is also consistent with the observation in E. coli that the Cas1 protein is dispensable for resistance to infection by phage $\lambda$ (Brouns et al., 2008).

About half of the CRISPR1 and CRISPR2 spacers exhibited similarity to the genome of phage M102. It has been shown for $S$. thermophilus that a correlation exists between resistance to phage infection and the number of spacers with sequence similarity to phage sequences (Bolotin et al., 2005). In the present study, only one pair, consisting of an infecting phage (M102) and a receptive strain (OMZ 381), was available for investigation. This limited material does not allow conclusions about the relationship between CRISPR content and phage resistance to be drawn. 
However, the high prevalence of M102-homologous spacer sequences among $S$. mutans strains suggests that $S$. mutans is frequently exposed to M102-like phages. Interestingly, most mismatches between homologous spacers and the M102 phage sequence affected the third base of the codon. This indicates that many different variants of M102 exist and could evade CRISPR-mediated resistance through silent or conservative mutations without affecting the function of phage-encoded proteins. Such a mechanism has been proposed for S. thermophilus, in which a single base pair change in the phage genome is sufficient to escape resistance caused by the CRISPR machinery (Barrangou et al., 2007; Deveau et al., 2008). This could represent a major driving force for the evolution of bacteriophage mutants.

Plasmids occur in about $5 \%$ of all S. mutans strains (Caufield et al., 1988). However, none of the CRISPR1 or CRISPR2 spacers showed sequence similarity to the previously identified S. mutans plasmids pLM7 and pUA140 (Zhou et al., 2001). This is surprising, since spacers with similarity to a plasmid from $S$. agalactiae were present in some strains. Equally unexpected, though not unprecedented, was the finding that two CRISPR1 spacers matched ORFs present in the S. mutans UA159 genome. There are no indications that these ORFs are located on genomic islands or are part of prophage sequences. Hence, it is not clear why spacers derived from these ORFs were incorporated. Speculatively, one could postulate that the CRISPR machinery occasionally confuses host DNA and host mRNA with invading nucleic acids and erroneously attacks the former. Alternatively, uptake of homologous chromosomal DNA of lysed S. mutans cells during natural competence might provoke capture of novel spacers.

In summary, this study demonstrates a high degree of variability in the sequences of CRISPR1 and CRISPR2 in $S$. mutans. A large fraction of CRISPR1 and CRISPR2 spacers have homology to M102, suggesting that the organism often encounters M102-like phages. Although the data presented indicate that acquired resistance to M102 infection cannot be explained by the function of the two identified CRISPR clusters alone, the emergence of phageresistant mutants following exposure to M102, as observed experimentally in vivo in a rat model, seems to be a frequent event.

\section{ACKNOWLEDGEMENTS}

I thank Verena Osterwalder for excellent technical assistance, Rudolf Schmid for performing the animal study leading to the isolation of OMZ 432, and Rudolf Gmür for critical reading of the manuscript. Roy Russell, University of Newcastle, Newcastle, UK, and Elaine Allan, Eastman Dental Institute, London, UK, are acknowledged for contributing some of the $S$. mutans strains.

\section{REFERENCES}

Ajdić, D., McShan, W. M., McLaughlin, R. E., Savić, G., Chang, J., Carson, M. B., Primeaux, C., Tian, R., Kenton, S. \& other authors
(2002). Genome sequence of Streptococcus mutans UA159, a cariogenic dental pathogen. Proc Natl Acad Sci U S A 99, 1443414439 .

Armau, E., Bousque, J. L., Boue, D. \& Tiraby, G. (1988). Isolation of lytic bacteriophages for Streptococcus mutans and Streptococcus sobrinus. J Dent Res 67, 121.

Bachrach, G., Leizerovici-Zigmond, M., Zlotkin, A., Naor, R. \& Steinberg, D. (2003). Bacteriophage isolation from human saliva. Lett Appl Microbiol 36, 50-53.

Barrangou, R., Fremaux, C., Deveau, H., Richards, M., Boyaval, P., Moineau, S., Romero, D. A. \& Horvath, P. (2007). CRISPR provides acquired resistance against viruses in prokaryotes. Science 315, 1709-1712.

Bolotin, A., Quinquis, B., Sorokin, A. \& Ehrlich, S. D. (2005). Clustered regularly interspaced short palindrome repeats (CRISPRs) have spacers of extrachromosomal origin. Microbiology 151, 2551-2561.

Brouns, S. J. J., Jore, M. M., Lundgren, M., Westra, E. R., Slijkhuis, R. J. H., Snijders, A. P. L., Dickman, M. J., Makarova, K. S., Koonin, E. V. \& other authors (2008). Small CRISPR RNAs guide antiviral defense in prokaryotes. Science 321, 960-964.

Carlsson, J. (1967). Presence of various types of non-haemolytic streptococci in dental plaque and in other sites of the oral cavity in man. Odontol Revy 18, 55-74.

Caufield, P. W., Ratanapridakul, K., Allen, D. N. \& Cutter, G. R. (1988). Plasmid-containing strains of Streptococcus mutans cluster within family and racial cohorts: implications for natural transmission. Infect Immun 56, 3216-3220.

Crooks, G. E., Hon, G., Chandonia, J.-M. \& Brenner, S. E. (2004). WebLogo: a sequence logo generator. Genome Res 14, 1188-1190.

Delisle, A. L. \& Rostkowski, C. A. (1993). Lytic bacteriophages of Streptococcus mutans. Curr Microbiol 27, 163-167.

Demuth, D. R., Lammey, M. S., Huck, M., Lally, E. T. \& Malamud, D. (1990). Comparison of Streptococcus mutans and Streptococcus sanguis receptors for human salivary agglutinin. Microb Pathog 9, 199-211.

de Stoppelaar, J. D., König, K. G., Plasschaert, A. J. M. \& Van der Hoeven, J. S. (1971). Decreased cariogenicity of a mutant of Streptococcus mutans. Arch Oral Biol 16, 971-975.

Deveau, H., Barrangou, R., Garneau, J. E., Labonte, J., Fremaux, C., Boyaval, P., Romero, D. A., Horvath, P. \& Moineau, S. (2008). Phage response to CRISPR-encoded resistance in Streptococcus thermophilus. J Bacteriol 190, 1390-1400.

Edwardsson, S. (1968). Characteristics of caries-inducing human streptococci resembling Streptococcus mutans. Arch Oral Biol 13, 637646.

Forde, A. \& Fitzgerald, G. F. (1999). Bacteriophage defence systems in lactic acid bacteria. Antonie Van Leeuwenhoek 76, 89-113.

Gibbons, R. J., Berman, K. S., Koettner, P. \& Kapsimalis, B. (1966). Dental caries and alveolar bone loss in gnotobiotic rats infected with capsule-forming streptococci of human origin. Arch Oral Biol 11, 549-560.

Glaser, P., Rusniok, C., Buchrieser, C., Chevalier, F., Frangeul, L., Msadek, T., Zouine, M., Couve, E., Lalioui, L. \& other authors (2002). Genome sequence of Streptococcus agalactiae, a pathogen causing invasive neonatal disease. Mol Microbiol 45, 1499-1513.

Grissa, I., Vergnaud, G. \& Pourcel, C. (2007). CRISPRFinder: a web tool to identify clustered regularly interspaced short palindromic repeats. Nucl Acids Res 35 (Web Server issue), W52-W57.

Grönroos, L. \& Alaluusua, S. (2000). Site-specific oral colonization of mutans streptococci detected by arbitrarily primed PCR fingerprinting. Caries Res 34, 474-480. 
Guggenheim, B. (1968). Streptococci of dental plaques. Caries Res 2, 147-163.

Haft, D. H., Selengut, J., Mongodin, E. F. \& Nelson, K. E. (2005). A guild of 45 CRISPR-associated (Cas) protein families and multiple CRISPR/ Cas subtypes exist in prokaryotic genomes. PLOS Comput Biol 1, e60.

Hamada, S. \& Ooshima, T. (1975). Inhibitory spectrum of a bacteriocinlike substance (mutacin) produced by some strains of Streptococcus mutans. J Dent Res 54, 140-145.

Hamada, S., Masuda, N. \& Kotani, S. (1980). Isolation and serotyping of Streptococcus mutans from teeth and feces of children. J Clin Microbiol 11, 314-318.

Horvath, P., Coûté-Monvoisin, A.-C., Romero, D. A., Boyaval, P., Fremaux, C. \& Barrangou, R. (2008a). Comparative analysis of CRISPR loci in lactic acid bacteria genomes. Int J Food Microbiol, doi:10.1016/j.ijfoodmicro.2008.05.030

Horvath, P., Romero, D. A., Coute-Monvoisin, A.-C., Richards, M., Deveau, H., Moineau, S., Boyaval, P., Fremaux, C. \& Barrangou, R. (2008b). Diversity, activity and evolution of CRISPR loci in Streptococcus thermophilus. J Bacteriol 190, 1401-1412.

Krasse, B. (1966). Human streptococci and experimental caries in hamsters. Arch Oral Biol 11, 429-436.

Kunin, V., Sorek, R. \& Hugenholtz, P. (2007). Evolutionary conservation of sequence and secondary structures in CRISPR repeats. Genome Biol 8, R61.

Makarova, K. S., Grishin, N. V., Shabalina, S. A., Wolf, Y. I. \& Koonin, E. V. (2006). A putative RNA-interference-based immune system in prokaryotes: computational analysis of the predicted enzymatic machinery, functional analogies with eukaryotic RNAi, and hypothetical mechanisms of action. Biol Direct 1, 7.

Marraffini, L. A. \& Sontheimer, E. J. (2008). CRISPR interference limits horizontal gene transfer in staphylococci by targeting DNA. Science 322, 1843-1845.

McShan, W. M., Ferretti, J. J., Karasawa, T., Suvorov, A. N., Lin, S., Qin, B., Jia, H., Kenton, S., Najar, F. \& other authors (2008). Genome sequence of a nephritogenic and highly transformable M49 strain of Streptococcus pyogenes. J Bacteriol 190, 7773-7785.
Paster, B. J., Boches, S. K., Galvin, J. L., Ericson, R. E., Lau, C. N., Levanos, V. A., Sahasrabudhe, A. \& Dewhirst, F. E. (2001). Bacterial diversity in human subgingival plaque. J Bacteriol 183, 3770-3783.

Perry, D. \& Kuramitsu, H. K. (1981). Genetic transformation of Streptococcus mutans. Infect Immun 32, 1295-1297.

Podbielski, A., Spellerberg, B., Woischnik, M., Pohl, B. \& Lütticken, R. (1996). Novel series of plasmid vectors for gene inactivation and expression analysis in group A streptococci (GAS). Gene 177, 137-147.

Shibata, Y., Ozaki, K., Seki, M., Kawato, T., Tanaka, H., Nakano, Y. \& Yamashita, Y. (2003). Analysis of loci required for determination of serotype antigenicity in Streptococcus mutans and its clinical utilization. J Clin Microbiol 41, 4107-4112.

Shibata, Y., Yamashita, Y. \& van der Ploeg, J. R. (2009). The serotype-specific glucose side chain of rhamnose-glucose polysaccharides is essential for adsorption of bacteriophage M102 to Streptococcus mutans. FEMS Microbiol Lett 294, 68-73.

Sorek, R., Kunin, V. \& Hugenholtz, P. (2008). CRISPR - a widespread system that provides acquired resistance against phages in bacteria and archaea. Nat Rev Microbiol 6, 181-186.

Thurnheer, T., Giertsen, E., Gmür, R. \& Guggenheim, B. (2008). Cariogenicity of soluble starch in oral in vitro biofilm and experimental rat caries studies: a comparison. J Appl Microbiol 105, 829-836.

van der Ploeg, J. R. (2007). Genome sequence of Streptococcus mutans bacteriophage M102. FEMS Microbiol Lett 275, 130-138.

Waterhouse, J. C. \& Russell, R. R. B. (2006). Dispensable genes and foreign DNA in Streptococcus mutans. Microbiology 152, 1777-1788.

Waterhouse, J. C., Swan, D. C. \& Russell, R. R. B. (2007). Comparative genome hybridization of Streptococcus mutans strains. Oral Microbiol Immunol 22, 103-110.

Zhou, X., Caufield, P. W., Li, Y. \& Qi, F. (2001). Complete nucleotide sequence and characterization of pUA140, a cryptic plasmid from Streptococcus mutans. Plasmid 46, 77-85.

Edited by: M. Kilian 